\title{
Analysis of Energy Conservation in Sensor Networks
}

\author{
Q.Gao ${ }^{1}$, K.J.Blow ${ }^{1}$, D.J.Holding ${ }^{1}$, Ian Marshall ${ }^{2}$ \\ ${ }^{1}$ Aston University, Aston Triangle, Birmingham, UK, B4 7ET, Tel: +44 121359 \\ 3611, Fax: +44 1213590156 \\ (qianggao@aston.ac.uk, k.j.blow@aston.ac.uk, d.j.holding@aston.ac.uk) \\ ${ }^{2}$ University of Kent, Canterbury, Kent, UK, CT2 7NF, Tel: +44 1227 827753, Fax: \\ +441227762811 \\ (i.w.marshall@kent.ac.uk)
}

\begin{abstract}
In this paper we use the Erlang theory to quantitatively analyse the trade offs between energy conservation and quality of service in an ad-hoc wireless sensor network. Nodes can be either sleeping, where no transmission or reception can occur, or awake where traffic is processed. Increasing the proportion of time spent in the sleeping state will decrease throughput and increase packet loss and delivery delay. However there is a complex relationship between sleeping time and energy consumption. Increasing the sleeping time does not always lead to an increase in the energy saved. We identify the energy consumption profile for various levels of sensor network activity and derive an optimum energy saving curve that provides a basis for the design of extended-life ad hoc wireless sensor networks.
\end{abstract}

Keywords: Sensor networks, Ad hoc networks, Energy efficient design, QoS, Erlang formula 


\section{Introduction}

Recent advances in micro-electro-mechanical systems (MEMS) technology, wireless communications and digital electronics have enabled the development of low-cost, low-power, multifunctional smart sensor nodes [1]. Smart sensor nodes are autonomous devices equipped with heavily integrated sensing, processing, and wireless communication capabilities [2][3]. When these nodes are networked together in an ad-hoc fashion, they form a sensor network. The nodes gather data via their sensors, process it locally or coordinate amongst neighbors and forward the information to the user or, in general, a data sink. Due to the node's limited transmission range, this forwarding mostly involves using multi-hop paths through other nodes [3]. A node in the network has essentially two different tasks: (1) sensing its environment and processing the information for onward transmission, and (2) forwarding traffic from other sensors as an intermediate relay in the multi-hop path.

The major design challenge for this type of network is to increase the operational lifetime of the sensors as much as possible [1][4]. Indeed, sensor nodes are miniature devices and operate on a tiny, non-replaceable battery. Energy efficiency is therefore the critical design constraint. Research can address two different perspectives of the energy problem: (1) an increase in battery capacity and (2) a decrease in the amount of energy consumed at the wireless terminal. The focus of battery technology research has been to increase battery power capacity while restricting the weight of the battery. However, unlike other areas of computer technology such as microchip design, battery technology has not experienced significant (compared to Moore's Law) advancement in the past 30 years. Therefore, unless a breakthrough occurs in battery technology, a goal of research should be to decrease the energy consumed in the wireless terminal [5].

In terms of energy consumption, the wireless exchange of data between nodes strongly dominates other node functions such as sensing and processing [6][7]. Moreover, actual radios consume power not only when sending and receiving data, but also when listening. Energy models have been developed [7][8] which show that the energy consumption ratio of listen:receive:send is about $1: 1: 1.5$. With this model, node listening time dominates energy consumption in light or moderate traffic scenarios. Significant energy savings are only obtainable by putting the node into a sleep mode when there is no traffic [4][9][10].

Given the importance of energy conservation in sensor networks we now describe a simple model that allows us to investigate a) the relationship between energy consumption and network traffic and $b$ ) the trade off between energy consumption and network performance. Our asynchronous queuing model looks rather like pure ALOHA with random periods of sleep although the local connectivity of nodes in a sensor network is quite different to the global connectivity of nodes in a simple ALOHA network. Recently, there has been some research on slotted ALOHA medium access control protocols for sensor networks [18][19][20]. The performance analysis of pure ALOHA algorithm provides a base level of performance for judging the effectiveness of the slotted ALOHA algorithm. Similarly, our simple model also provides a benchmark against which the performance of more complex sleep synchronisation protocols can be judged.

\section{Description of sensor node}

We consider a network of wireless sensor nodes where each node consists of a transmitter and receiver together with some sensing device. For our purposes the sensing device is a source of information flow local to the sensor node. We will assume that the sensor node is kept as simple as possible in order to enable mass deployment. Thus, we assume that a single antenna is available and that the node can 
transmit or receive but not both simultaneously. Each sensor node will accept information from other nodes for onward transmission. There will also be special 'sink' nodes in the network where information terminates, these will not be explicitly modelled here as we are only interested in the relationships between local and transit traffic and how these affect the performance of the network. Since nodes will have limited computational and memory resources we will explicitly model the queuing of packets in the node.

The four possible states of the nodes are shown in Figure 1. A node is said to be 'active' when it is transmitting or receiving information and 'idle' when it is listening (i.e. listening but not actually receiving data) or asleep (i.e. not listening to the outside world). These four states have different levels of energy consumption, as we will discuss in the next section. Transitions between these states can occur naturally, such as the change from listening to receiving when data arrives from a neighbouring node, or as a result of some internal decision, such as the scheduling algorithm used to decide when to change from listening to sleeping and vice versa.

\section{Energy-conserving algorithm}

To reach our low energy target, we can let radios sleep most of the time and yet let them awaken precisely when they need to transmit or receive data. Unfortunately, current radio technology does not easily allow a radio to be awakened upon request. Hence, a radio must wake up periodically, see if anyone wants to talk to it, and, if not, go back to sleep. The simple energy-conserving algorithm that we will analyse will now be described.

Nodes are in one of the following states: sleeping, listening, sending or receiving. The state transition diagram is shown in Figure 1. We use the term idle to describe the sleeping and listening states and the term active to describe the sending or receiving states.

Initially nodes start out in the sleeping state. When sleeping, the radio is off and therefore not consuming power. (Although the radio is off, sensors or other low power parts of the node may be on.) In this state the radio remains turned off for time $T_{s}$ and then undergoes a transition to listening. If, however, while a node is sleeping data to be transmitted is generated then it changes to the active transmit state and starts sending the data. During the listening state if neighboring nodes try to transfer packets via the node, or if data needs to be sent, the node changes to the appropriate active state. Otherwise it returns to the sleeping state after time $T_{1}$. When in an active state a node either sends or receives data. After all data transmission has finished, the node changes to an idle state and begins alternately sleeping and listening according to some pre-defined pattern.

The algorithm that turns off the radio is targeted to improve power consumption. However turning off the radio has implications for network performance such as reduced throughput, added latency and possibly more packet loss compared to the communication protocols without sleeping patterns. Here our objective is to characterize the trade-off and enable optimal control of the sleep/listen pattern in future networks.

We must point out that here we do not consider the synchronization of sleep schedule among the nodes as discussed in [14] where they listen at the same time and go to sleep at the same time. Though synchronization might reduce the additional latency it will increase the chance of overhearing traffic when a node picks up packets that are destined to other nodes and it will also increase the control packet overhead (synchronization packets). These are two additional sources of energy consumption and also increase the complexity of the algorithm. It should be noticed that the randomly occurring sensor information traffic is a potential interruption to the 
synchronization and to avoid this would require storage of information in the queue until the next waking period. This might well increase the required queue lengths in the nodes. So we consider the simplest algorithm in which each node chooses its own sleep schedule independently. This asynchronous algorithm may in some cases increase the probability of network connection. This is because long-range synchronisation involves some network overhead. Methods based on local synchronisation can lead to islands of synchronisation where nodes within an island are synchronised but different islands are not [16][17]. This is illustrated in Figure 2 where a node in island 1 wants to transmit data to a node in island 5. Recall that in a sensor network the radio range will generally be smaller than the network size and so global synchronization to a central clock is difficult to guarantee. In this simple example the nodes in island 1 and island 5 cannot communicate directly. The probability of connection from the node in island 1 to the node in island 5 in an asynchronous sleep schedule is larger than in a synchronous sleep schedule because there are more opportunities to find one of the nodes in islands 2, 3 or 4 awake. In the extreme case where the islands wake-up periods do not overlap then no communication will be possible. This problem can be avoided if the sensor nodes move or there is some level of noise. However, when the network is sufficiently large the finite propagation speed of the information will limit the achievable global synchronization or at best lead to synchronization on extremely long timescales. Also, the performance analysis of a simple asynchronous algorithm provides a base level of performance for judging the effectiveness of synchronous or other more complex algorithms in the future.

\section{Theoretical analysis}

Here we use a similar method to the Erlang formula in telephone systems [25] to calculate the effects of throughput, packet loss and delay induced by turning off the radio. Let us consider that node 1 needs to transfer data to node 2. Suppose that A is the traffic in Erlang that needs to be transferred by node1. It may be local data or data needing to be forwarded from neighboring nodes. The number of packets waiting in the queue is $\mathrm{x}$ and the number of packets in onward transmission is $\mathrm{s}$. Let $[\mathrm{x}, \mathrm{s}]$ denote the state of the sending node 1 when $\mathrm{x}$ packets are in the waiting queue and s packets are in onward transmission; $u(x, s)$ the probability that a packet begins to be transmitted after a packet arrives at state $[\mathrm{x}, \mathrm{s}] ; v(x, s)$ the probability that, after a packet finishes transmission, another packet begins to be transmitted. They are expressed as the following respectively

$$
u(x, s)=\left\{\begin{array}{c}
T,(s=0) \\
0,(s=1)
\end{array} \quad v(x, s)=\left\{\begin{array}{c}
T,(x \geq 1, s=1) \\
0,(x=0 \text { or } s=0)
\end{array}\right.\right.
$$

where $s \in\{0,1\} ; 0 \leq x \leq K$ and $\mathrm{K}$ is the length of waiting queue. $\mathrm{T}$ is the connection probability from node 1 to node 2 and is determined by the sleeping time, listening time of receiving node 2 and the traffic between node 1 and node 2 which we will discuss in detail later.

Assuming that the traffic can be described by Poisson distributed arrival times and negative exponential holding times (the holding time corresponds to the packet length) we can obtain general equations of statistical equilibrium [13]. The assumption of Poisson statistics is questionable given the nature of the anticipated applications of sensor networks. We might expect more bursty traffic described by fractal statistics to occur. However, at present there is no experimental evidence available to resolve this issue. The use of Poisson statistics allows us a) to build an 
analytic model of traffic dependent energy consumption and b) to provide a base model against which future numerical simulations can be judged.

Let $p(x, s)$ be the probability of the state $[\mathrm{x}, \mathrm{s}]$. Consider a very short interval time $d t$. By the assumption that packets occur individually, i.e. in accordance with the Poisson distribution, the probability of a packet arrival is $A d t$ (terms in higher powers of $d t$ are neglected). By the assumption that the traffic packets have a negative-exponential holding-time distribution, the probability that a packet finishes transmission is $s d t$. For the statistical equilibrium assumption that $p(x, s)$ is independent of the time, this implies that the state $p(x, s)$ is created as often as it is destroyed otherwise $p(x, s)$ would change with time. Therefore the general equations of statistical equilibrium are as follows:

$$
\begin{aligned}
& (A+s) p \quad(x, s)=A[1-u \quad(x-1, s)] p \quad(x-1, s)+A u \quad(x, s-1) p \quad(x, s-1 \\
& +(s+1)[1-v(x, s+1)] p \quad(x, s+1)+s v(x+1, s) p \quad(x+1, s)
\end{aligned}
$$

The left-hand side of the equation represents the rate at which the system leaves the state $[\mathrm{x}, \mathrm{s}]$ due to the packet arrival rate $A p(x, s)$ and packet finishing transit rate $s p(x, s)$. The first term of the right represents the rate at which the system leaves the state [x$1, \mathrm{~s}]$ and enters $[\mathrm{x}, \mathrm{s}]$, the second term represents the rate at which it leaves [x,s-1] and enters $[\mathrm{x}, \mathrm{s}]$, the third and the forth terms represent the rate at which it leaves $[\mathrm{x}, \mathrm{s}+1]$ or $[\mathrm{x}+1, \mathrm{~s}]$ and enters $[\mathrm{x}, \mathrm{s}]$ respectively.

To give the solution we have the further standard assumption [14]: transition probabilities from state $[\mathrm{x}-1, \mathrm{~s}]$ to state $[\mathrm{x}, \mathrm{s}]$ and from $[\mathrm{x}, \mathrm{s}-1]$ to $[\mathrm{x}-1, \mathrm{~s}]$ are the same as those from $[\mathrm{x}, \mathrm{s}]$ to $[\mathrm{x}-1, \mathrm{~s}]$ and from $[\mathrm{x}, \mathrm{s}]$ to $[\mathrm{x}, \mathrm{s}-1]$, respectively. The following expressions are then obtained for the probability of the state $[\mathrm{x}, \mathrm{s}]$ that $\mathrm{x}$ packets are in the queue and s packets are in transit.

$$
\begin{gathered}
p \quad(x, 0)=\frac{A^{x}(1-T)^{x}}{T^{x}} p(0,0) \\
p(x, 1)=A^{x+1} T p \quad(0,0)+\sum_{i=1}^{x} \frac{A^{x+1}(1-T)^{i}}{T^{i-1}} p(0,0)
\end{gathered}
$$

Using the fact that $p \quad(K+1, s)=0$ we have

If $T<\frac{1}{2}$ and $0 \leq A<\frac{T}{1-T}$ or $\frac{T}{1-T}<A<1$

$1 / p(0,0)$

$$
\begin{aligned}
& =\frac{1-\left[\frac{A(1-T)}{T}\right]^{K+1}}{1-\frac{A(1-T)}{T}}+\frac{T A\left(1-A^{K+1}\right)}{1-A}+\frac{A^{2}(1-T)\left\{1-\left[\frac{A(1}{T}\right.\right.}{(1-A)\left[1-\frac{A(1-T}{T}\right.} \\
& -\frac{A^{K+2}(1-T)\left[1-\left(\frac{1-T}{T}\right)^{K}\right]}{(1-A)\left(1-\frac{1-T}{T}\right)}
\end{aligned}
$$

If $T<\frac{1}{2}$ and $A=\frac{T}{1-T}$ 
$=K+1+\frac{T A\left(1-A^{K+1}\right)}{1-A}+\frac{K A^{2}(1-T)}{1-A}-\frac{A^{K+2}(1-T)\left[1-\left(\frac{1-T}{T}\right)^{K}\right]}{(1-A)\left(1-\frac{1-T}{T}\right)}$

If $T=\frac{1}{2}$ and $0 \leq A<1$

$1 / p(0,0)$
$=\frac{1-\left[\frac{A(1-T)}{T}\right]^{K+1}}{1-\frac{A(1-T)}{T}}+\frac{T A\left(1-A^{K+1}\right)}{1-A}+\frac{A^{2}(1-T)\left\{1-\left[\frac{A(1-T)}{T}\right]\right.}{(1-A)\left[1-\frac{A(1-T)}{T}\right]}$

If $T>\frac{1}{2}$ and $0 \leq A<1$

$\begin{aligned} 1 / p(0,0) & 1-\left[\frac{A(1-T)}{T}\right]^{K+1} \\ = & \frac{T A\left(1-A^{K+1}\right)}{1-\frac{A(1-T)}{T}}+\frac{A^{2}(1-T)\left\{1-\left[\frac{A(1-T)}{T}\right]^{K}\right\}}{(1-A)\left[1-\frac{A(1-T)}{T}\right]}-\frac{A^{K+2}(1-}{(1-A)(}\end{aligned}$

The probability of packet loss is

$$
L=p(K, 1)+p \quad(K, 0)
$$

The carried traffic is

$$
A^{\prime}=A[1-p \quad(K, 1)-p \quad(K, 0)]
$$

The average delay time, that is the waiting time averaged over the carried traffic, is

$$
D=\frac{\bar{q}}{A^{\prime}}
$$

where the average number of waiting packets is

$$
\bar{q}=\sum_{x=1}^{K} x p \quad(x, 1)+\sum_{x=1}^{K} x p \quad(x, 0)
$$

\section{Connection Probability T}

In an ad-hoc network each node is, in general, surrounded by a dynamic collection of other nodes. Each node also performs a dual function of sending out data collected locally and relaying data sent from other nodes as shown in Figure 3. While transmission of local data will only involve the use of the transmitter, relaying network traffic will involve the use of both the receiver and the transmitter. Since our simple nodes cannot perform transmit and receive simultaneously we need to account for the mutual blocking of these two processes. When a node has some data needing to be sent out, it should try to establish a connection first. We denote the connection probability by $\mathrm{T}$. We assume that the sending operation has a higher priority than the receiving operation. Then the sending node can connect to the receiving node when there is no packet in the waiting queue of the receiving node and the receiving node is also in the listening state. If we also assume that every node is equivalent, from a 
traffic carrying perspective, in the whole network then we have the following formula for the connection probability

$$
T=T_{0} p \quad(0,0)
$$

where $T_{0}=\frac{T_{l}}{T_{s}+T_{l}}$ represents the sleeping time factor of the receiving node, $T_{l}$ and $T_{s}$ are the listening time and sleeping time respectively. When there is heavy traffic in the network there will always be some packets in the waiting queue of the receiving node, which will keep trying to send the packets out. Under these conditions, the probability of the receiver being available is very low. Our calculations based on this assumption, that transmission has a higher priority than receiving, confirm that the throughput in the network is very low under heavy traffic conditions.

Here we consider the alternative prioritisation, that the receiving operation has higher priority than the sending operation. The sending node can successfully connect to the receiving node when there is no packet in the waiting queue of the receiving node and it is in listening state or when there are some packets in the waiting queue but the receiving node is not actually in the process of sending packets. In this case the formula for the connection probability becomes

$$
T=T_{0} p(0,0)+\left(1-p \quad(0,0)-A^{\prime}\right)
$$

where the first term is the contribution due to being in the listening state and the second term is the contribution when there are some packets in the queue but the receiving node is not actually sending information. Note that $A$ ' is the traffic that is actually carried by the node and that the connection probability given by equation (14) is always larger than (13). From equations (5-8), (10) and (14) we can selfconsistently calculate the connection probability $\mathrm{T}$ and the other variables.

\section{Trade-off between Energy Conserving and QoS}

Quality of Service (QoS) is an important consideration in any communications network. It can be measured in many ways depending on the nature of the applications deployed. In our case, we will use packet delay or packet loss as measures of QoS.

In order to save the energy in sensor networks we turn off the radio when there is no data to transfer. However it will reduce the network throughput and increase packet loss and delay. This technique trades off energy savings versus QoS of sensor networks. We can deduce the relationships, which are shown in the following figures from the formulas above.

In Figure 4, the packet loss rate $\mathrm{L}$ is plotted versus the offered traffic, A, on one node. The length of its waiting queue is $K=5$. We observe that for higher values of the sleeping time $T_{s}$, that is the smaller $T_{0}$, the higher the packet loss becomes. Indeed, as $A$ approaches 1 these curves will diverge in the normal way as the offered traffic is close to the maximum capacity.

Figure 5 shows the carried traffic $A$ ' as a function of the offered traffic $A$. The peak of each curve corresponds to the maximum throughput. We observe that for higher values of the sleeping time $\mathrm{T}_{\mathrm{s}}$, that is the smaller $T_{0}$, the throughput in the network is lower, as we would expect. The carried traffic curve in the absence of the blocking effect (i.e. if two radio cards are used, one for sending and one for receiving) is also shown in this figure.

In Figure 6, the packet delay $\mathrm{D}$ is plotted versus the amount of traffic $A$. We observe that the packet delay increases with increased sleeping time. In other words, conserving energy leads to a reduction in network QoS. We have also observed that longer queues lead to longer packet delays with an associated reduction in packet loss in the normal way, which is not shown in the figures. 
We now take the energy consumption ratios to be (note that energy consumption is zero in the sleeping state) listen:receive:send $=1: 1: \mathrm{b}$. The total energy consumption of one node can then be expressed as

$E=A^{\prime}+\left(1-A^{\prime}\right) p(0,0) T_{0}+b\left(1-A^{\prime}\right)[1-p(0,0)]$

The first term $A^{\prime}$ is the energy consumption for data receiving. The second term $\left(1-A^{\prime}\right) p(0,0) T_{0}$ is the energy consumption of periodically waking up for listening when the node is idle, that is when it is neither sending nor receiving traffic. The last term $b\left(1-A^{\prime}\right)[1-p(0,0)]$ is the energy consumption when the node is in sending state. There are two parts in this term: one is the energy consumption for the actual process of sending packets $b A^{\prime}$, which we call the sending consumption; the other is the energy consumption for establishing a connection before the sending operation $b\left(1-A^{\prime}\right)[1-p(0,0)]-b A^{\prime}$, which is called the connecting consumption.

Figure 7 shows the energy consumption of one node for data receiving, data sending, connection establishing, listening and the total energy consumption when $T=0.3, K=5, b=1.5$ and $T=0.5, K=5, b=1.5$. We can see that the difference in energy consumption, for establishing connections, between $T=0.3$ and $T=0.5$ is much bigger than the differences in energy consumption for other contributions such as listening, sending and receiving data. This is the main reason for the complex relationship between energy saved and sleeping time. A longer sleeping time does not always lead to an increase in energy saving. In some cases we cannot even save energy by adding a sleeping schedule into the communication protocol.

Figure 8 shows the surface of energy consumption $\mathrm{E}(K=5, b=1.5)$ versus the amount of traffic A and the sleeping time factor variable $T_{0}$. We can save the energy from the area of the energy consumption surface under the energy consumption plane $(\mathrm{T}=1.0)$ when there is no sleeping schedule in the radio. The maximum energy saving curve (i.e. minimum energy consumption curve) is shown in Figure 8 as well.

In Figure 9, the energy consumption normalized to the maximum energy consumption is plotted versus the amount of traffic $A$ when, $T_{0}=0.1$ and $T_{0}=0.5$ for the energy consumption ratio 1:1.05:1.4 [21]. For comparison, simulation results from recent work [22] based on numerical simulations are shown as point values. The agreement is excellent given that the only fitting parameter required was the scaling between traffic levels in the numerics and the analytic model. Our model clearly captures the essential elements of the network properties which allow the overall network performance to be predicted. Indeed, the numerical model also allowed for nonPoissonian statistics and the level of agreement might well suggest that this plays a small part in determining the overall network performance.

Suppose the sensor network had a perfectly synchronised sleep schedule. Here we don't consider how this happens or how much network traffic would be necessary to make it happen. We can calculate the performance of such a network by setting $T_{0}=1$ in equation (14). The connection probability is independent of the sleeping time under synchronized conditions. The packet delivery delay for any amount of sleeping time is given by the curve corresponding to $T_{0}=1.0$ in figure 6 . This is a result of the receiver model assumption that the transition to the sleeping state can occur when the node is idle. Thus the node is active for a much larger fraction of time than the sleeping schedule would predict. If we keep the connection probability factor $T_{0}=$ 1.0 in equation (14) but use $T_{0}=0.5$ in equation (15) we can calculate the energy consumption when the node spends half of the time in the sleeping state under synchronized conditions. Figure 10 shows the comparison between the energy consumption under synchronized and unsynchronized conditions when $T_{0}=0.5$. The relatively modest energy saving that is achieved through synchronisation would be 
reduced by the network traffic needed to maintain synchronisation. Further detailed study of synchronization algorithms is therefore required in order to demonstrate useful energy savings from their application.

The radio transceiver TR1000 from RF Monolithics, Inc is widely used in developing sensor nodes. When using OOK modulation at a transmission rate of $19.2 \mathrm{kbps}$ the power consumption is $13.5 \mathrm{~mW}, 24.75 \mathrm{~mW}$ and $0.015 \mathrm{~mW}$ in receiving, transmitting and sleep respectively. There is no difference between listening and receiving in this radio transceiver model [23]. When it provides a transmission rate of $2.4 \mathrm{kbps}$ the power consumption in receiving, listening, transmitting and sleep is $12.5 \mathrm{~mW}$, $12.36 \mathrm{~mW}, 14.88 \mathrm{~mW}$ and $0.016 \mathrm{~mW}$ respectively [24]. There is no significant change of our results for these representative power numbers.

\section{Conclusions}

In wireless sensor networks, where energy efficiency is the key design challenge, the energy consumption is typically dominated by the node's communication subsystem. It can only be reduced significantly by transitioning the radio to a sleep state when there is no traffic needing to be transferred. However increasing the sleeping time will reduce throughput and increase packet loss and delivery delay. We have quantitatively analysed the relationship between energy consumption and sleeping time and the relationship between QoS and sleeping time. Increasing the proportion of time spent in the sleeping state will decrease the QoS but does not always lead to an increase in the energy saved. We have also shown that, where use of the transmitter and use of the receiver are mutually exclusive then network performance is improved by giving higher priority to the receiver.

Our analysis shows that the relationship between sleeping time, energy consumption and QoS is complex and subtle and that detailed analysis and design is therefore required to achieve good energy saving and an acceptable QoS in ad hoc sensor networks. We have identified the energy consumption profile for various levels of sensor network activity and also derived an optimum energy saving curve that provides a basis for the design of extended-life ad hoc wireless sensor networks.

\section{References}

[1] I. F. Akyildiz, W. Su, Y. Sankarasubramaniam, and E. Cayirci, "Wireless Sensor Networks: A Survey", Computer Networks, 38 393-422, 2002

[2] K. Sohrabi, J. Gao, V. Ailawadhi and G. Pottie, "Protocols for self-organization of a wireless sensor network," IEEE Personal Communications Magazine, 7 16-27, 2000 [3] L. Clare, G. Pottie and J. Agre, "Self-organizing distributed sensor networks," SPIE - The International Society for Optical Engineering, Orlando, FL, 229-237, April 1999

[4] C. E. Jones, K. M. Sivalingam, P. Agrawal, and J. C. Chen, "A survey of energy efficient network protocols for wireless networks", Wireless Networks, 7 343-358, 2001

[5] Lettieri, P. and M. srivastava, "Advances in wireless terminals", IEEE Personal communications, 6 6-18, 1999

[6] D. Estrin and R. Govindan, "Next century challenges: scalable coordination in sensor networks," MobiCom 1999, Seattle, USA, 263-270, 1999

[7] A. Savvides, C. C. Han and M. Srivastava, "Dynamic fine-grained localization in ad-hoc networks of sensors," MobiCom 2001, Rome, Italy, 166-179, 2001

[8] M. Stemm and R. H. Katz, "Measuring and reducing energy consumption of network interfaces in hand-held devices", IEICE Transactions on Communications, E80-B 1125-1131, 1997 
[9] J. M. Rabaey, M. J. Ammer, J. L. da Silva Jr., D. Patel and S. Roundy, "PicoRadio Supports Ad Hoc Ultra Low-Power Wireless Networking," IEEE Computer, 33 42-8, 2000

[10] C. Schurgers, V. Tsiatsis and M.B. Srivastava, "STEM: Topology management for energy efficient sensor networks", IEEE Aerospace Conference, 2001

[11] Y. Xu, J. Heidemann, and D. Estrin, "Adaptive energy-conserving routing for multihop ad-hoc networks", USC/ISI Research Report 527, 2000

[12] C. S. Raghavendra, S. Singh, "PAMAS--Power Aware Multi-Access Protocol with signaling for Adhoc Networks", Computer Communications Review, July 1998

[13] D. Bear, "Principles of Telecommunication. Traffic Engineering", Peter Peregrinus Ltd. 1988

[14] M. H. Thierer, "Delay systems wich limited accessibility", preprints of technical papers, $5^{\text {th }}$ International Teletraffic Congress, New York, 203-213, 1967

[15] W. Ye, J. Heidemann and Deborah Estrin, "An Energy-Efficient MAC Protocol for Wireless Sensor Networks", the Proceedings of the 21st International Annual Joint Conference of the IEEE Computer and Communications Societies (INFOCOM 2002), New York, NY, USA, 2002

[16] I. Wokoma, I. Liabotis, O. Prnjat, L. Sacks, I. Marshall, "A Weakly Coupled Adaptive Gossip Protocol for Application Level Active Networks", IEEE 3rd International Workshop on Policies for Distributed Systems and Networks - Policy 2002, Monterey, CA, USA, 2002

[17] R. E. Mirollo, S. H. Strogatz, "Synchronization of pulse coupled biological oscillators", SIAM J. Applied Mathematics, 1990

[18] R. Simon and E. Farrugia, Topology-transparent support for sensor networks,in: Proc. of 1st European Workshop on Wireless Sensor Networks (LCNS 2920), Berlin, Germany, 122-137, 2004

[19] P. Venkitasubramaniam, S. Adireddy and L. Tong, Opportunistic ALOHA and Cross-Layer Design for Sensor Networks, in: Proc. of IEEE Military Comm. Conf., Boston, USA, 123-128, 2003

[20] R. Zheng, J. Hou and L. Sha, Asynchronous Wakeup for Ad Hoc Networks, in: Proc. of MobiHoc 2003, Annapolis, Maryland, USA, 35-35, 2003

[21] Q. Gao, D.J. Holding, Y. Peng and K.J. Blow, Energy Efficiency Design Challenge in Sensor Networks, in: Proc. of LCS 2002, London, UK, 69-72, 2002

[22] S. Raghumanshi and A. Mishra, An Approach Towards Improved Energyefficiency in Wireless Sensor Nets, in: Proc. of IEEE RAWCON 2003, Boston USA, 225-228, 2003

[23] C. Schurgers, V. Tsiatsis, S. Ganeriwal and M. B. Srivastava, Optimizing Sensor Networks in the Energy-Latency-Density Design Space, IEEE Transactions on Mobile Computing, 1(1) 70-80, 2002

[24] W. Ye, J. Heidemann and D. Estrin, Medium Access Control with Coordinated, Adaptive Sleeping for Wireless Sensor Networks, Technical Report ISI-TR-567, USC/Information Sciences Institute, 2003

[25] J.E. Flood, Telecommunications, Switching, Traffic and Networks, Longman 1994 


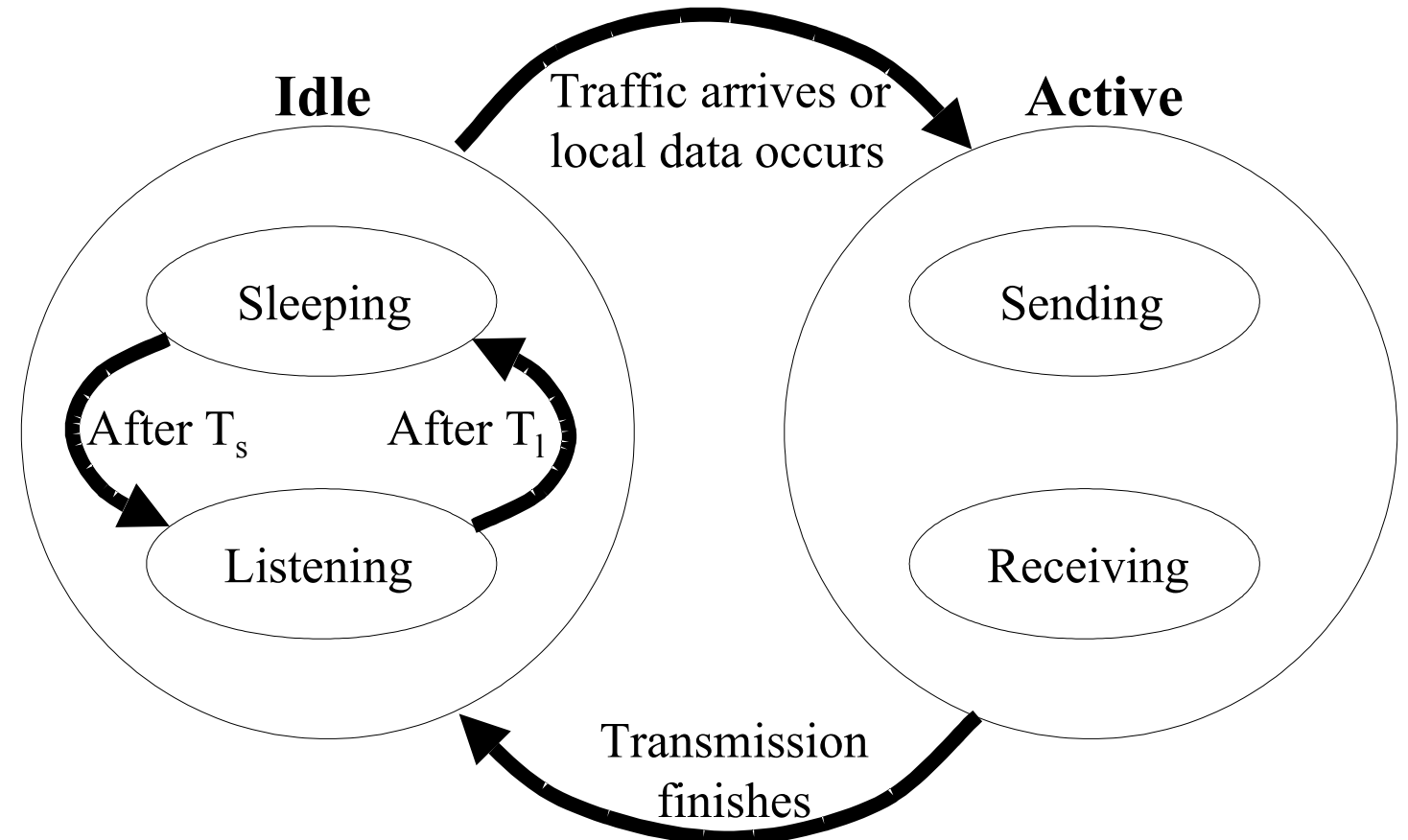

Figure 1: State diagram of the model sensor node. 


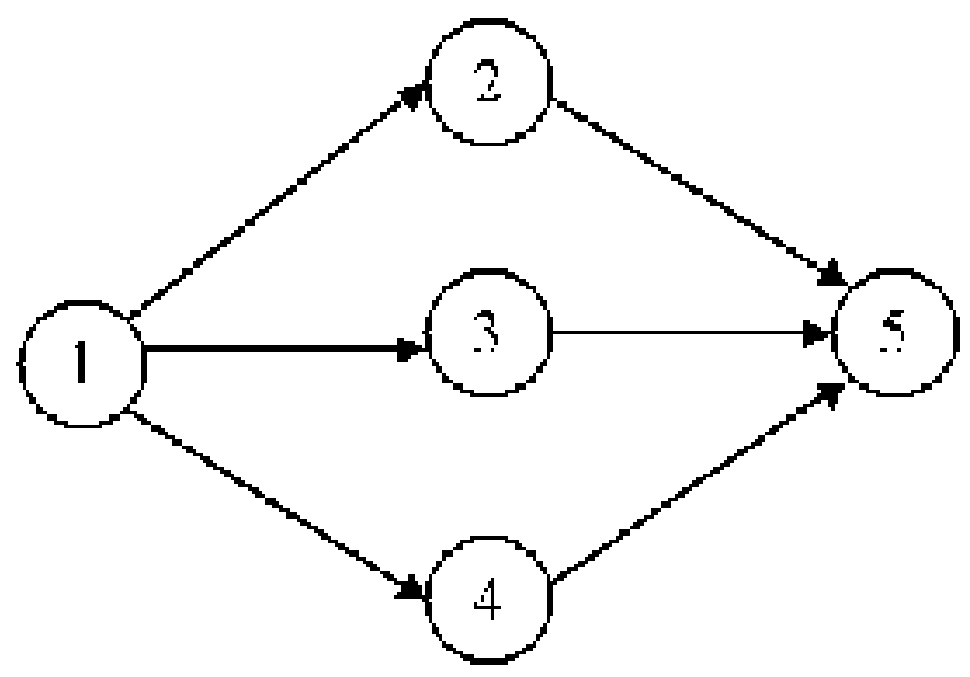

Figure 2: Illustration of a partially synchronised network. Each island, labeled 1-5, consists of a set of nodes that have achieved local synchronisation. Since the islands are unsynchronised with each other, this leads to a lower probability of a network path between island 1 and island 5 compared to the asynchronous case. 


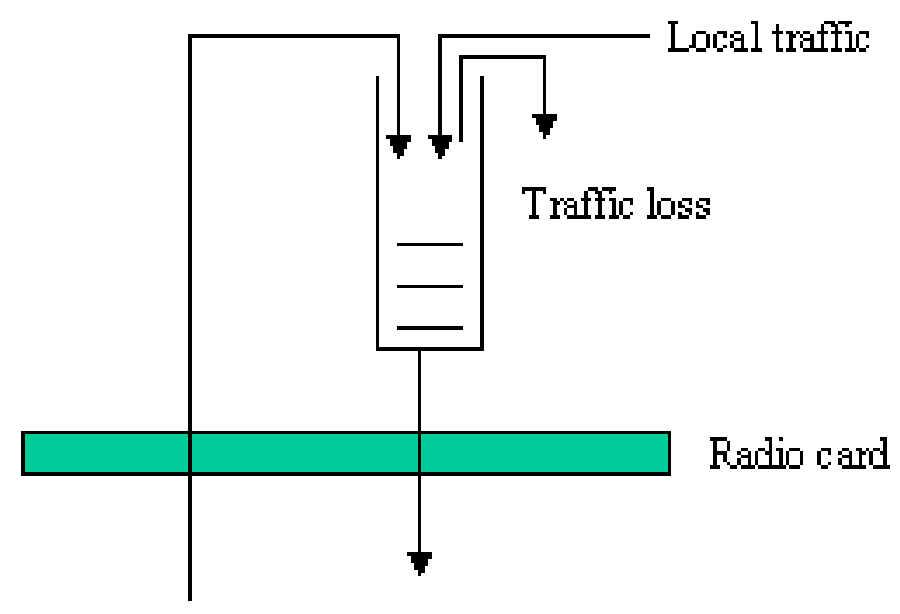

Forwarded traffic Camied traffic

Figure 3: Diagram illustrating the traffic flows in the node, note that the sending operation and the receiving operation may block each other. 


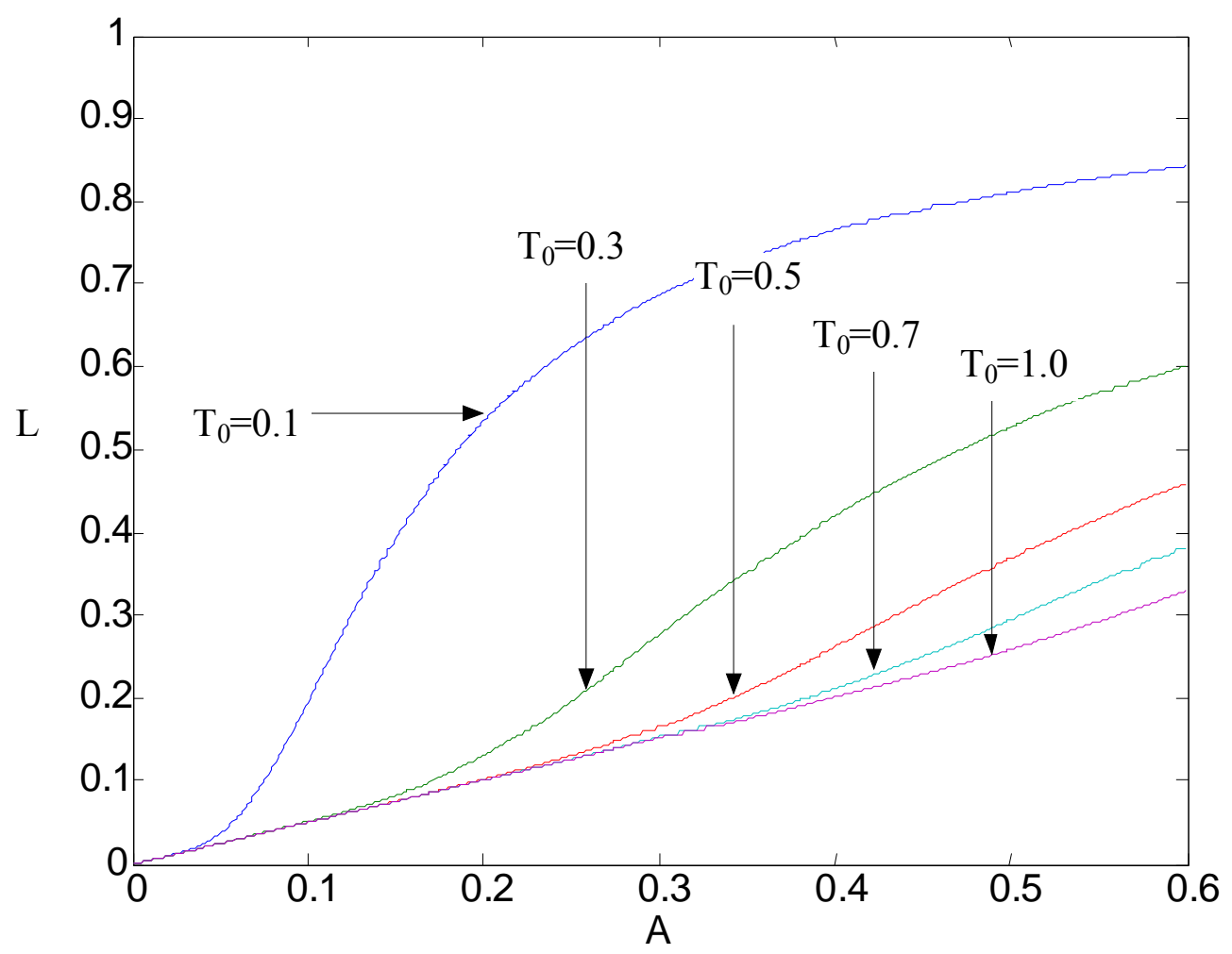

Figure 4: Packet loss ratio as a function of traffic for the energy-conserving algorithm. 


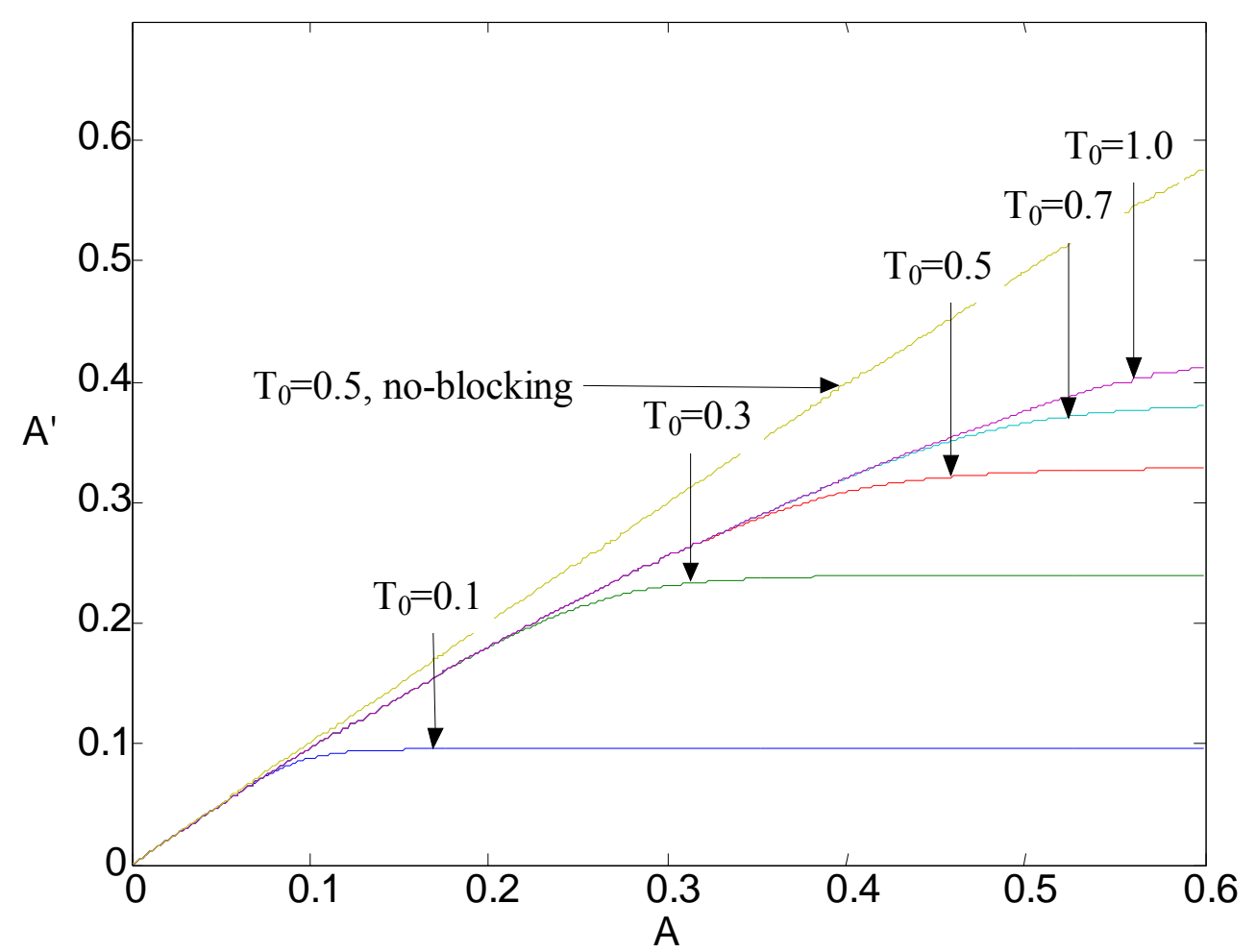

Figure 5: Carried traffic as a function of offered traffic for the energy-conserving algorithm. 


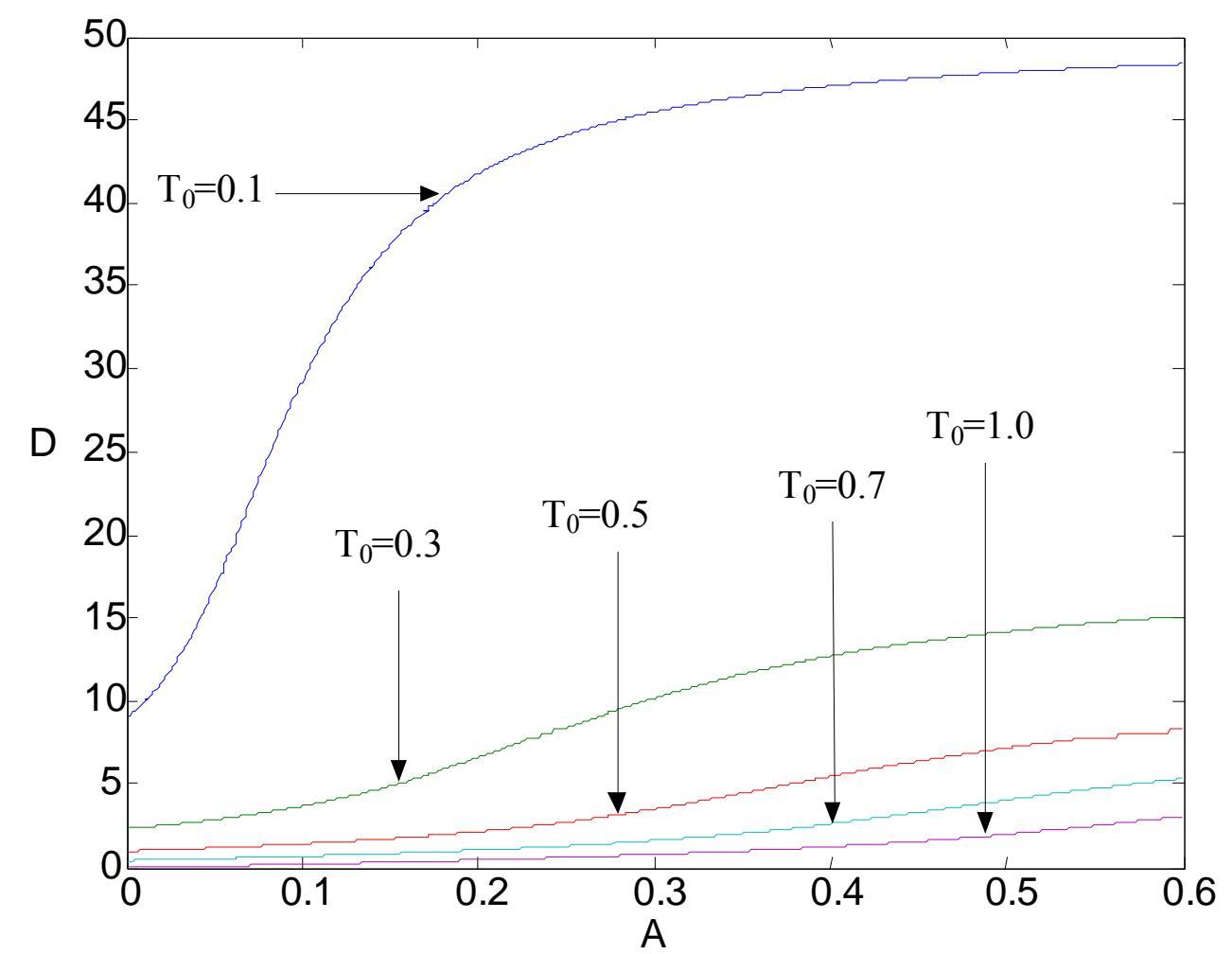

Figure 6: Packet delivery delay as a function of traffic for the energy-conserving algorithm. 


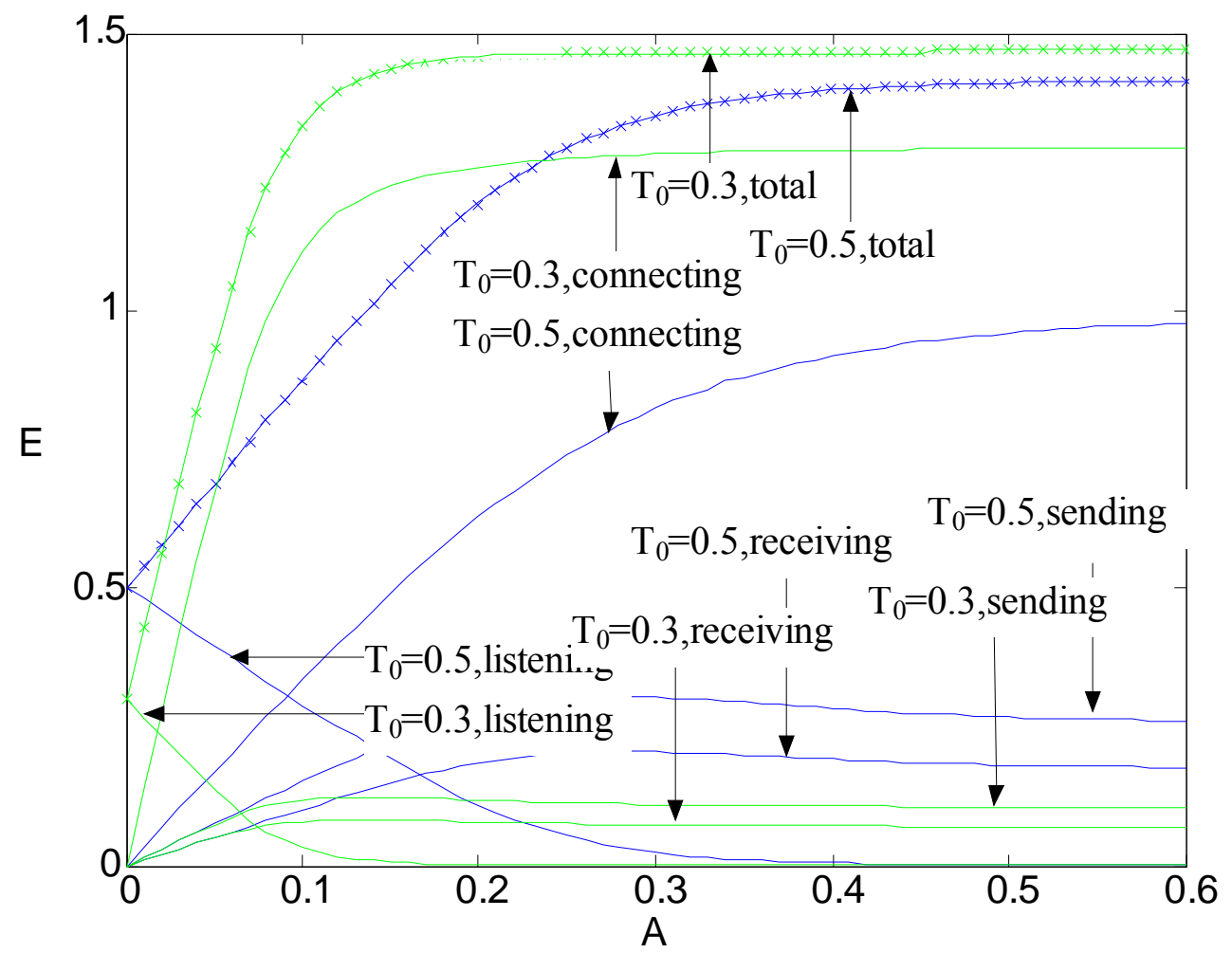

Figure 7: Energy consumption breakdown when $\mathrm{T}_{0}=0.3$ and $\mathrm{T}_{0}=0.5$. 


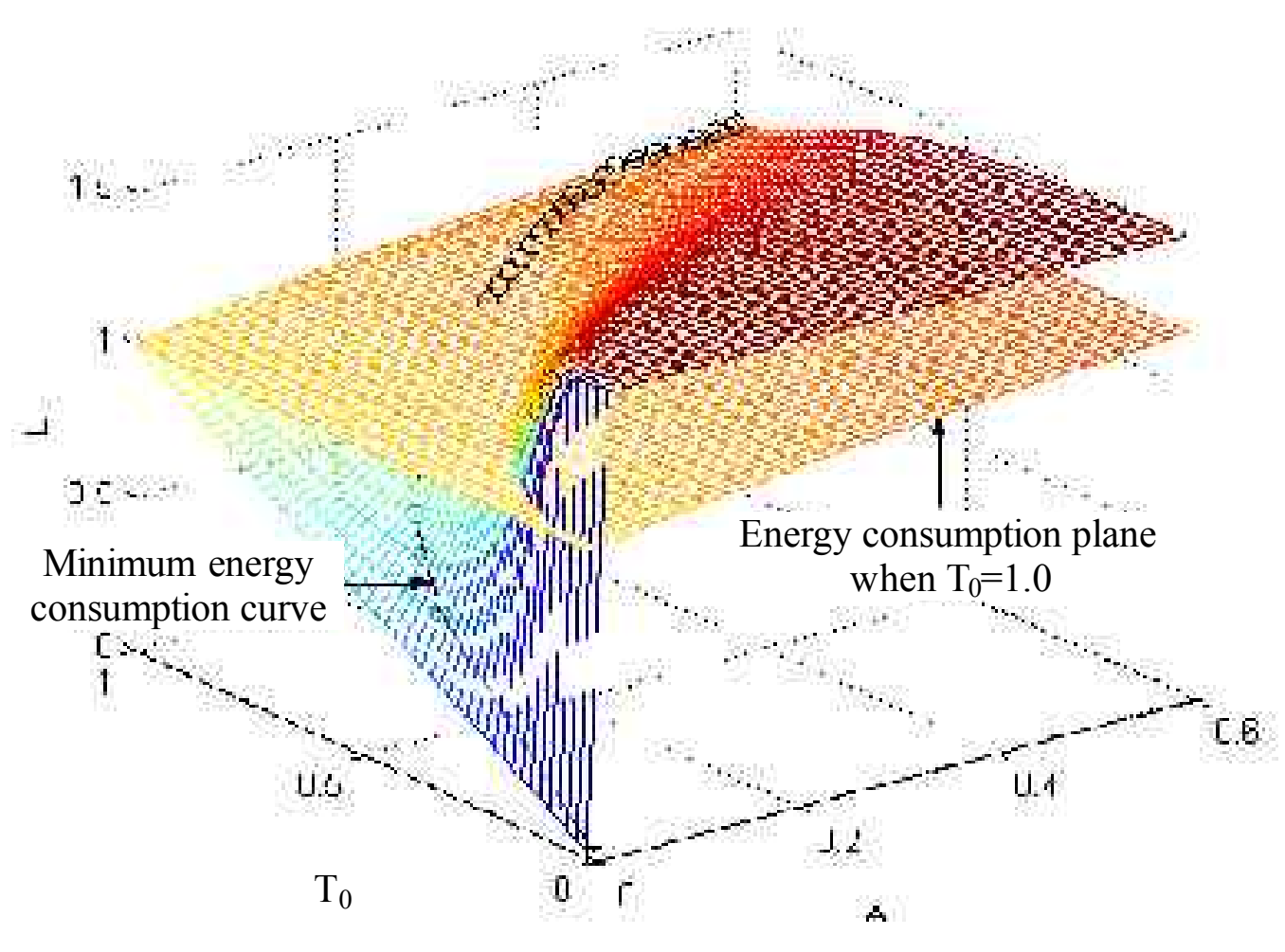

Figure 8: Energy consumption surface and minimum energy consumption curve. 


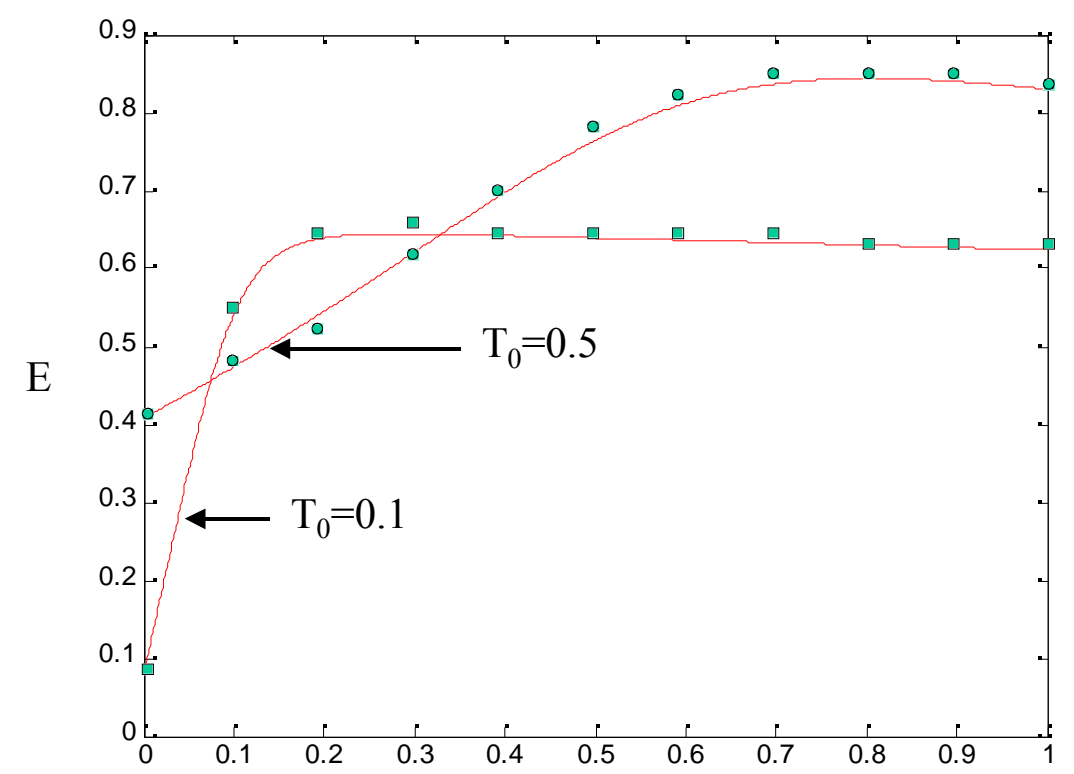

A

Figure 9: Energy consumption comparison between the theoretical model (solid lines) and recent simulation results (points) [22]. 


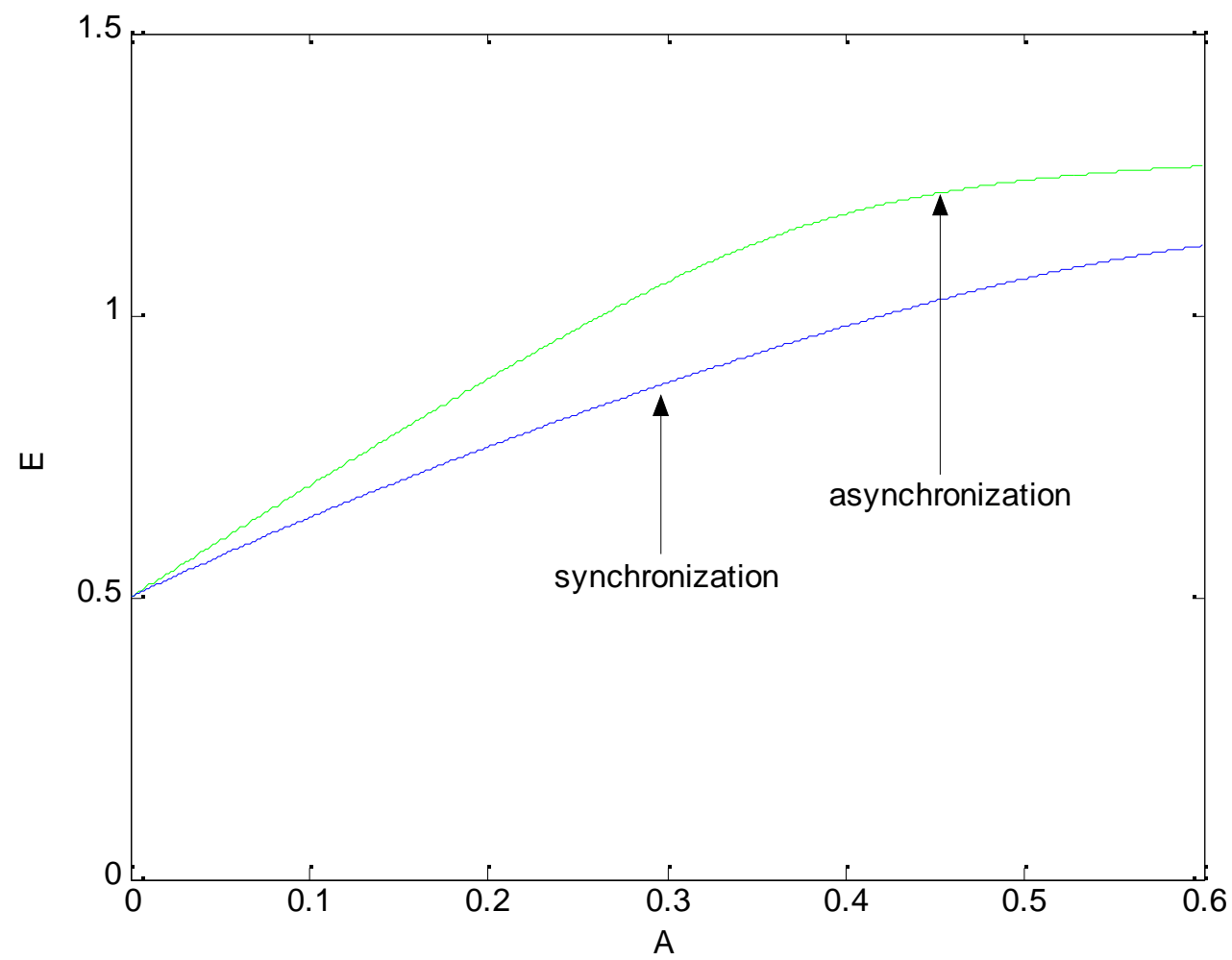

Figure 10: Energy consumption as a function of traffic when $T_{0}=0.5$ under synchronized and unsynchronized conditions. 